\title{
Quand l'enquêteur se met à table
}

Cuisine et gastronomie dans le « polar méditerranéen » et le « nouveau roman policier grec »

When the Investigator Seats to Eat: Cooking and Gastronomy in the Mediterranean Thrillers and the "New Greek Detective Novels"

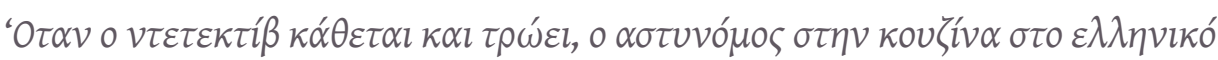

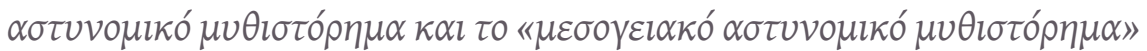

\section{Loïc Marcou}

\section{(2) OpenEdition} Journals

Édition électronique

URL : https://journals.openedition.org/ceb/6839

DOI : $10.4000 /$ ceb.6839

ISSN : 2261-4184

Éditeur

INALCO

Édition imprimée

ISBN : 978-2-85831-230-6

ISSN : 0290-7402

Référence électronique

Loïc Marcou, "Quand l'enquêteur se met à table », Cahiers balkaniques [En ligne], Hors-série | 2016, mis en ligne le 08 mars 2016, consulté le 06 juillet 2021. URL : http://journals.openedition.org/ceb/6839 ; DOI : https://doi.org/10.4000/ceb.6839

Ce document a été généré automatiquement le 6 juillet 2021.

\section{c) (7) (5)}

Cahiers balkaniques est mis à disposition selon les termes de la Licence Creative Commons Attribution - Pas d'Utilisation Commerciale 4.0 International. 


\title{
Quand l'enquêteur se met à table
}

\author{
Cuisine et gastronomie dans le «polar méditerranéen » et le « nouveau \\ roman policier grec » \\ When the Investigator Seats to Eat: Cooking and Gastronomy in the \\ Mediterranean Thrillers and the "New Greek Detective Novels"

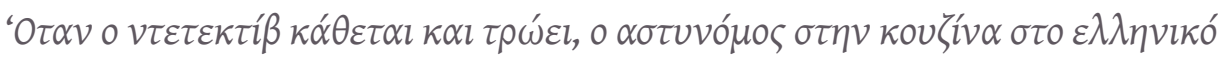

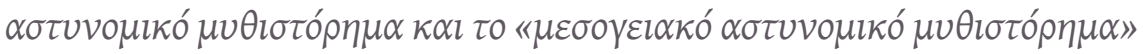

Loïc Marcou

1 La cuisine et la gastronomie occupent une place importante dans la littérature policière en général et dans le roman policier grec en particulier. Sans faire un mauvais jeu de mots, on peut dire que, dans tout " polar » qui se respecte, l'enquêteur policier ne se contente pas de faire en sorte que le suspect se mette à table ${ }^{1}$; il aime également manger à une bonne table. En d'autres termes, si son travail consiste à faire cracher le morceau au coupable, l'enquêteur aime aussi manger un morceau, non pas tant pour satisfaire un besoin élémentaire que pour s'adonner à un plaisir terrestre qu'il place audessus de tous les autres. On se rappelle que le commissaire Maigret de Simenon raffole des petits plats concoctés par sa femme Louise ${ }^{2}$. Quant à l'astynomos Bekas, le personnage sériel de Maris - dont on sait qu'il est directement inspiré du policier français ${ }^{3}$-, il affectionne les mets préparés par son épouse Evanthia.

2 On pourrait s'interroger au préalable sur la présence de la cuisine (c'est-à-dire l'art ou la manière d'apprêter les aliments) et de la gastronomie (autrement dit l'amour de la bonne chère) dans le genre policier. Il existe selon nous quatre raisons à cela.

3 En présentant l'enquêteur comme un être doté d'un solide appétit, l'auteur de récits policiers donne tout d'abord à ce dernier une touche d'humanité. Celle-ci est primordiale parce qu'elle renforce l'illusion référentielle (l'enquêteur apparaît tout à la fois comme un personnage bien et bon vivant ${ }^{4}$ ) et parce qu'elle facilite l'identification du lecteur au protagoniste. En étant présenté comme une solide fourchette, l'enquêteur apparaît d'emblée, en effet, comme un personnage humain, voire sympathique, auquel le lecteur peut plus facilement s'identifier. Il ne fait guère de doute, à cet égard, que ce dernier préférera un fin gourmet s'adonnant, en marge de ses investigations, au plaisir 
de la bonne chère à un être anorexique concentré sur ses seules enquêtes. Si le lecteur ne s'identifie pas nécessairement à Nero Wolfe ${ }^{5}$ (le personnage obèse né de l'imagination de Rex Stout), il sera en revanche sensible au charme suranné du commissaire Maigret qui, de retour du Quai des Orfèvres, se demande chaque soir quel plat sa femme a bien pu lui mitonner : bœuf miroton, blanquette de veau, fricandeau à l'oseille, tarte aux mirabelles, etc. D'autre part, dans un genre qui tourne par essence autour de la vie et de la mort et qui charrie parfois des questions d'ordre métaphysique, la gastronomie apparaît aussi comme l'un des rares plaisirs permettant à l'enquêteur de dissiper l'angoisse du néant. Plus qu'un art de vivre, l'amour de la bonne chère serait donc un art de survivre. C'est ce qu'a justement remarqué Maryse Vuillermet dans un article consacré à l'œuvre policière de Jean-Claude $\mathrm{Izzo}^{6}$. On se souvient que dans tel passage de Chourmo (1996), l'écrivain marseillais fait dire à Fabio Montale :

J'aime ça manger, mais c'est pire quand j'ai des ennuis, et pire encore quand je côtoie la mort. J'ai besoin d'ingurgiter des aliments, légumes, viandes, poissons, desserts ou friandises. De me laisser envahir par leurs saveurs. Je n'avais rien trouvé de mieux pour réfuter la mort. M'en préserver. La bonne cuisine et les bons vins. Comme un art de survivre ${ }^{7}$.

4 La présence de la gastronomie dans le roman policier tient en outre au fait qu'en fin limier, l'enquêteur est toujours attiré par l'odeur de la chair: celle du cadavre de la victime qui réclame justice ou celle de l'animal mort qu'il mange plus qu'à son tour. De manière plus prosaïque, on pourrait enfin affirmer que l'amour de la bonne chère, souvent érigé en profession de foi par l'enquêteur policier, est avant tout celui de son créateur. On se souvient que Georges Simenon présentait ses plats de prédilection dans ses romans, que Manuel Vázquez Montalbán fut critique gastronomique avant de devenir auteur de récits policiers, que Patricia Cornwell a créé un personnage d'enquêteur gastronome ${ }^{8}$ en raison de sa passion pour l'art culinaire, etc ${ }^{9}$. De manière générale, le lien quasi consubstantiel entre cuisine et roman policier est corroboré par les innombrables ouvrages consacrés à l'univers gastronomique de tel enquêteur ou de tel auteur de "polars ». Parmi les livres de recettes inspirés de romans policiers, on citera: Crèmes et Châtiments ${ }^{10}$, la Sauce était presque parfaite ${ }^{11}$, Alimentaire, mon cher Watson $!^{12}$, le Livre de cuisine de la Série Noire ${ }^{13}$, les Recettes de Carvalho ${ }^{14}$, Simenon et Maigret passent à table $e^{15}$, etc.

Dans le cadre de cet article, nous ne nous étendrons pas sur la place de la cuisine et de la gastronomie dans le genre policier en général. Nous nous focaliserons sur les raisons de sa présence dans le nouveau roman policier grec en faisant un rapide rapprochement avec ce que l'on présente parfois comme un nouveau sous-genre: le "polar méditerranéen ${ }^{16}$ ». Nous employons à dessein l'expression : "nouveau roman

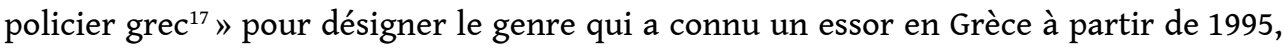
date de parution simultanée de la Partie perdu $e^{18} \mathrm{~d}$ 'Andréas Apostolidis et de Journal de la nuit $^{19}$ de Pétros Markaris. Sur bien des points sur lesquels, faute de temps, nous ne nous étendrons pas ici, le nouveau roman policier grec -j'entends celui de la triade d'auteurs sériels: Apostolidis (1953-), Markaris (1937-), Martinidis (1946-) - est en rupture avec le premier récit policier hellénique, celui né après-guerre sous la plume de Yannis Maris (1916-1979). De manière générale, il s'agira de montrer quel rôle symbolique la cuisine et la gastronomie jouent dans le roman policier grec contemporain. 


\section{Cuisine et gastronomie dans le « polar méditerranéen »}

6 On a coutume de dire que la cuisine est une des mythologies - au sens barthésien du terme - véhiculées par le «polar méditerranéen ${ }^{20}$ », celui de Manuel Vázquez Montalbán (1939-2003), Andrea Camilleri (1925-) et Jean-Claude Izzo (1945-2000). On sait que Pepe Carvalho, le personnage récurrent de Montalbán, ne cesse de faire bonne chère en marge de ses enquêtes. On se souvient que Salvo Montalbano, le héros sériel de Camilleri, est un fin gourmet qui raffole des petits plats mitonnés par sa bonne (Adelina), par sa compagne génoise (Livia) ou par les tenanciers des multiples trattorie qu'il fréquente au cours de ses enquêtes. On se rappelle enfin que Fabio Montale refoule la peur de la mort en mangeant et en buvant avec les gens simples qui constituent son cercle d'amis dans « la trilogie marseillaise ${ }^{21}$ » d'Izzo.

7 Le roman policier grec contemporain accorde lui aussi une place à la cuisine, mais il renouvelle le rôle dévolu à la gastronomie dans le " polar méditerranéen ». Expliquonsnous. Dans le roman policier de l'aire méditerranéenne, en présentant un enquêteur amateur de bonne chère, l'auteur en profite généralement pour faire un éloge de la cuisine méridionale sous toutes ses formes. Prenons l'exemple de Pepe Carvalho, le personnage récurrent de Montalbán. On se souvient qu'en marge de ses investigations, le détective privé catalan aime faire bombance en solitaire ou avec ses bons amis. Or, ces agapes, qui constituent un défilé de plats originaires de tous les coins de la Méditerranée (la Catalogne, bien sûr, mais aussi les Canaries, les Baléares, la Provence ou le Mezzogiorno), rendent un hommage à l'art culinaire d'Europe méridionale. De même qu'il existe une koinè linguistique, il existerait en effet, selon Carvalho, une koinè gastronomique qui coïnciderait peu ou prou avec l'aire méditerranéenne. Écoutons la profession de foi du détective catalan dans Tatouage (1974), le deuxième roman policier de la série de Vázquez Montalbán :

Au cours des dix dernières années, la Catalogne avait vu naître plus de dix mille restaurants animés des mêmes intentions : fournir à la clientèle les miracles de simplicité qu'offre la cuisine rurale catalane. Mais à l'heure de vérité, le pain à la tomate, merveille d'imagination qui dépasse la pizza en saveur et en simplicité, se révélait n'être qu'une pâte mal cuite, ramollie encore par la sauce tomate en boîte. [...]. Carvalho se surprit à donner une conférence sur les racines gastronomiques de l'humanité aux dames qui lui tenaient compagnie, bouche bée : [...] « De même qu'il $\mathrm{y}$ a une koinè linguistique qui nous permet de situer l'origine des langues germaniques dans l'indo-européen, il existe une koinè gastronomique, dont une des manifestations scientifiquement décelable est le pain à la tomate, proche parent de la pizza, mais plus facile à réaliser ${ }^{22}$.

8 Comme on le voit, Pepe Carvalho n'est pas un gastronome régionaliste ou nationaliste, bref un adepte de la seule cuisine catalane ou espagnole. Bien au contraire, il se fait le héraut d'une gastronomie qui unirait tous les terroirs de la Méditerranée selon un credo bien ancré : «plats alimentaires de tous les pays, unissez-vous!». Comment ne pas voir dans cet éloge du pain à la tomate un hommage rendu à l'ensemble de la culture gastronomique méditerranéenne et non l'expression d'un quelconque régionalisme culinaire? 


\section{Cuisine et gastronomie dans le nouveau roman policier grec (Apostolidis, Martinidis)}

Dans le nouveau roman policier grec, la cuisine et la gastronomie ne jouent pas a priori un rôle aussi important que dans le "polar méditerranéen ". De manière générale, on peut affirmer qu'il n'existe aucune mise en valeur de l'art culinaire méditerranéen ou grec dans le roman policier hellénique. C'est ce que nous allons montrer en évoquant tout d'abord les romans policiers d'Andréas Apostolidis et Pétros Martinidis puis ceux de Pétros Markaris, que l'on peut à bon droit considérer comme le chef de file du «nouveau roman policier grec ».

En premier lieu, on constatera que la cuisine est totalement absente des "polars " d'Andréas Apostolidis, sauf peut-être dans Crimes à la pension Apollon (2000) où l'avocat Ikonomou ${ }^{23}$ doit élucider deux meurtres perpétrés dans un lieu de villégiature. Dans cette nouvelle inspirée de l'univers romanesque d'Agatha Christie, l'enquête en lieu clos se déroule souvent au moment des repas. L'avocat Ikonomou, qui joue les apprentis enquêteurs, sonde en effet les différents suspects autour de la table de la pension. Mais la commensalité évoquée dans le récit d'Apostolidis ne célèbre ni les saveurs ni les odeurs de la cuisine méditerranéenne. De fait, on ne décèle ici aucune " promotion » de la cuisine grecque traditionnelle (féta, salade paysanne, kokoretsi, youvetsi, ouzo ou raki $i^{24}$ ), mais plutôt celle d'une cuisine internationale agrémentée d'alcools étrangers (Irish coffee $e^{25}$, Bloody Mary ${ }^{26}$, thé au cognac ${ }^{27}$, whisky $\left.{ }^{28}\right)^{29}$. Ce choix n'est pas anodin puisqu'Apostolidis décrit une atmosphère cosmopolite : on se souvient que la pension "Apollon» est tenue par un Grec assisté de son compagnon anglais et qu'elle est fréquentée par des Grecs de la diaspora mêlés à divers membres de l'extrême gauche européenne.

11 Dans l'œuvre policière de Pétros Martinidis, la cuisine en général et la commensalité en particulier jouent un rôle autrement plus important. On peut dire qu'elles ont partie liée avec l'atmosphère de mondanité qui imprègne les romans policiers de l'écrivain salonicien et qu'elles s'inscrivent dans la thématique de prédilection de l'auteur: l'imposture sous toutes ses formes. On rappellera que Pétros Martinidis décrit des microcosmes dans ses deux séries policières : le petit monde de l'université grecque et ses guerres larvées dans la tétralogie Quatre meurtres à l'universitée ${ }^{30}$ et l'univers des koultouriarides (artistes et auteurs dramatiques, éditeurs, journalistes) dans la trilogie Morts théâtrales ${ }^{31}$. On comprend donc que les repas occupent une place de choix dans ces mondes miniatures où les relations sociales et l'urbanité jouent par nature un rôle prépondérant. Dans Meurtres en série, le narrateur est souvent invité à des réceptions organisées par des mandarins de l'université Aristote. Dans ces agapes, selon les dires $\mathrm{du}$ narrateur, la bonne chère permet de supporter l'insupportable, à savoir ces universitaires qui ne cessent d'étaler leur culture creuse tout en mangeant et en buvant :

Nous nous mêlons à la foule. Autour de nous, l'on n'aperçoit que des femmes en toilette et des hommes en cravate, des bijoux rutilants et des sourires étincelants. [...] À l'entrée du pilotis, près de l'entrée du bâtiment principal, nous retrouvons Nikos Vlachopoulos. Assis sur un muret, il mange méthodiquement une assiette remplie de canapés au saumon et au caviar. Près de lui, son verre de vin est déjà vide. [...] Nous gagnons immédiatement le buffet, avec Nikos qui a déjà vidé son verre et qui s'empresse de se resservir. La boisson permet effectivement de 
supporter le petit monde des professeurs d'université. Surtout quand ils sont aussi nombreux ${ }^{32}$.

La cuisine tient également une place de choix dans Reflets du destin ${ }^{33}$. On se souvient que dans ce récit, le jeune Olmézoglou est invité par son père à assister à un colloque sur la dramaturgie des Atrides à Delphes. En marge des débats, Alexis participe à de nombreux repas réunissant tout le "gratin » de la recherche européenne en études théâtrales. Ce sera pour lui l'occasion de faire montre de ses talents d'orateur. Car s'il est beaucoup question du boire et du manger dans les romans de Martinidis, il est à remarquer que, bien souvent, les personnages pérorent tout en mangeant selon une tradition qui remonte à la plus haute Antiquité :

Peut-être est-ce là un abrégé de l'intelligentsia grecque : des gens qui, entre des bouchées et des gorgées successives, mélangent tout - le sexe et la philosophie, la politique et l'art, l'humour et la Bible - sans la moindre hésitation et sans craindre de tacher de graisse les sujets dont ils traitent pêle-mêle. [...]. La bombance collective et le bavardage sont inscrits dans nos gènes depuis les banquets de l'époque classique ${ }^{34}$.

Mais Martinidis ne se contente pas d'évoquer la tradition du symposion. Si la cuisine est présente dans ses romans, c'est parce qu'elle participe de la satire des différents microcosmes effectuée par l'écrivain salonicien. Ainsi, les intellectuels mis en scène dans Reflets du destin sont des adeptes des nourritures spirituelles, mais ils sont loin de dédaigner les nourritures terrestres, surtout quand ils ont la possibilité de manger «à l'œil » :

Il me faut beaucoup de retenue pour ne pas me jeter sur la nourriture avec la boulimie des autres convives, $\mathrm{y}$ compris mon illustre père. Ce sont pour la plupart des intellectuels avec une "énorme culture ", mais ils confirment l'adage latin primum vivere, deinde philosophari - d'abord la subsistance, la pensée vient après. Cela suppose que l'on ne parle pas la bouche pleine. Au milieu des services qui se succèdent, des généreuses libations d'huile dans les salades et des remplissages réitérés des verres, le bourdonnement des conversations ne cesse de s'amplifier ${ }^{35}$.

$14 \mathrm{Au}$ vu de ces quelques citations, on peut donc constater que la cuisine joue un rôle différent de celui qui lui est généralement dévolu dans le "polar méditerranéen ". Ainsi, les protagonistes de Martinidis ne sont pas de fins gourmets à la recherche de saveurs méridionales, à l'image de Pepe Carvalho ou de Salvo Montalbano. Ils ne mangent pas non plus pour refouler la peur de la mort comme Fabio Montale. Chez Martinidis, la gastronomie n'est pas, en effet, érigée en art de vivre ou de survivre. Quant à la commensalité, elle n'est pas synonyme de fraternité ou d'ouverture à l'autre : elle est une façade, un simulacre de relation, une pure convention sociale. En somme, elle s'inscrit dans une sociabilité théâtrale (une "hypocrisie" au sens étymologique du terme) où il est avant tout question d'étaler un vernis de culture tout en mangeant et en buvant. Difficile donc de discerner dans l'œuvre de l'écrivain salonicien une influence quelconque du " polar méditerranéen ».

\section{Cuisine et gastronomie dans l'œuvre policière de Pétros Markaris}

Dans le nouveau roman policier hellénique, c'est surtout chez Pétros Markaris que la cuisine occupe la place la plus importante. A priori, le commissaire Charitos ne fait pourtant pas figure de fin gourmet ou de gros mangeur. Le personnage récurrent de 
l'écrivain athénien est en effet très absorbé par ses tâches professionnelles et il a parfois la tentation de manger sur le pouce. Ainsi, on le voit avaler un croissant (sous cellophane) en guise de petit-déjeuner et parfois un simple sandwich pour tout repas de midi. Dans Journal de la nuit, le roman qui ouvre la série des aventures du commissaire Charitos, le rituel du croissant matinal joue cependant un rôle particulier dans la mesure où il met l'accent sur les petites manies de l'enquêteur. Le charme de l'illusion référentielle fonctionnant immédiatement, le lecteur voit ainsi apparaître sous ses yeux un homme en chair et en os, en l'occurrence un policier vieux jeu qui ne renoncerait pour rien au monde à son habitude du petit-déjeuner au bureau. Dans l'extrait suivant, Costas Charitos évoque la confrontation muette se déroulant quotidiennement entre le brigadier Nollis et lui-même. Jouant au jeu du chat et de la souris avec son subordonné, le policier athénien lui confie en permanence des tâches subalternes. Parmi celles-ci, le jeune policier se voit contraint d'apporter tous les jours le café et le croissant à son " patron »:

Dès le premier jour [Thanassis] m'a fait clairement comprendre qu'il était un crétin, et je l'en ai estimé d'autant plus. [...] Je jette un coup d'œil sur le bureau où je ne vois ni café ni croissant. C'est là, en effet, la seule mission régulière de Thanassis : m'apporter chaque matin un café et un croissant. Je lève la tête d'un air interrogateur. «Et alors, Thanassis, où est donc passé mon petit-déjeuner ce matin? Tu l'as oublié ? $»^{36}$

Si la cuisine renforce l'illusion référentielle en présentant un personnage de chair et d'os, on peut dire qu'elle joue surtout un rôle symbolique dans l'œuvre policière de Markaris. Elle permet en effet à l'auteur d'aborder des questions liées directement à la nature de la société grecque contemporaine : le rôle de la tradition, la répartition des tâches domestiques dans le couple et, de manière générale, la place de l'homme et de la femme dans une société méridionale. Expliquons-nous.

Dans l'œuvre policière de Markaris, force est d'abord de constater que la cuisine (dans sa double acception: celle de lieu destiné à la préparation des aliments et celle de manière de les apprêter) est le domaine réservé de la femme (Adriani) ${ }^{37}$ et non de l'homme (Costas). Dans le couple de petits-bourgeois grecs inspiré de la paire de personnages créée après-guerre par Yannis Maris (Georges et Evanthia Békas), c'est en effet la femme qui prépare le repas, en aucun cas l'homme. S'il apparaît comme un héritier en droite ligne du " patriarche ${ }^{38}$ », Markaris a cependant alloué à la cuisine une fonction qui n'apparaissait pas dans l'œuvre de Maris : celle de sceller la réconciliation, d'apaiser les différends, d'aplanir les tensions entre époux. Rappelons qu'au dénouement de Journal de la nuit, le couple Charitos se réconcilie autour d'un plat de légumes farcis (yemista) tendrement mitonnés par Adriani à son mari :

Un plat de tomates farcies est posé sur la table de la cuisine. Je saisis tout de suite le message. C'est la façon pour Adriani de me dire qu'il est temps de faire la paix. Cela nous est resté de notre première scène de ménage. Nous étions alors jeunes mariés et nous supportions mal de ne pas nous parler, mais nous tenions bon; pour mesurer chacun la force de résistance de l'autre. Jusqu'à ce qu'un jour Adriani me fasse des tomates farcies. Elle savait que c'était mon plat préféré, mais elle ne m'en avait jamais fait. [...] Depuis, cela nous est resté. Chaque fois qu'elle veut que nous nous réconciliions, elle me mitonne des tomates farcies ${ }^{39}$.

Si, dans le premier " polar » de Markaris, la cuisine ritualise une scène de réconciliation en vertu d'un principe connu (cuisiner pour quelqu'un, c'est l'aimer), elle est loin de se limiter à cette simple fonction dans les autres romans de la série. De manière générale, on peut dire qu'elle est un des éléments - ou l'un des ingrédients, pour filer la 
métaphore culinaire - de l'enquête menée par Markaris sur la société grecque contemporaine, une société bouleversée par les différents problèmes induits par la mondialisation puis par la "crise grecque», une société ballottée entre tradition et (post-) modernité, etc. Qu'on en juge d'après ces deux extraits qui me semblent particulièrement significatifs :

Quand je suis entré dans la police nous mangions des petits pains au sésame, nous essuyions le bureau d'un revers de la main pour balayer les graines de sésame et, face à nous, se tenait un Dimos, un Ménios ou un Lambros, assassin, truand ou vulgaire voleur à la tire. [...] Autrefois, nous avions du pain au sésame et des Grecs ; maintenant, nous avons des croissants et des Albanais ${ }^{40}$.

19 Autrefois, on servait l'ouzo avec des amuse-gueules : des canapés à la tomate fraîche avec une olive, une petite tranche de saucisse, un ou deux anchois. La quantité de horsd'œuvre augmentait avec le nombre de verres. Au dixième ouzo, on vous apportait carrément une assiette. Aujourd'hui, que l'on boive de l'ouzo, du whisky ou du cognac, c'est du pareil au même. On vous jette un petit bol de cacahuètes et de noisettes pour toute pâture ${ }^{41}$.

Comme on peut l'observer au vu de ces deux extraits dominés par la dialectique passéprésent, Pétros Markaris se livre, par le truchement de la métaphore culinaire, à une réflexion sur la nature de la société hellénique contemporaine. En mettant en scène un enquêteur nostalgique de la cuisine grecque d'antan, l'auteur athénien en profite pour dépeindre un pays écartelé entre tradition et modernité et victime d'un malaise identitaire. Au tournant du Xxi ${ }^{e}$ siècle, la Grèce de Markaris apparaît en effet comme un pays en perte de repères. Traditionnellement terre d'émigration, elle est devenue une terre d'immigration ${ }^{42}$ (avant de redevenir aujourd'hui un pays d'émigration). Autrefois xénophile, elle est en passe de devenir xénophobe ${ }^{43}$. Naguère dominée par le triptyque : "Patrie-Religion-Famille ${ }^{44}$ ", elle voit progressivement ces valeurs se diluer dans l'océan d'un monde globalisé. D'où un vertige identitaire parfaitement rendu par le plus célèbre des auteurs policiers grecs actuels au moyen - entre autres - de la métaphore culinaire.

\section{Conclusion}

21 En guise de conclusion, on peut affirmer que le nouveau roman policier grec relève difficilement de la catégorie du " polar méditerranéen ». Contrairement à Montalbán, Camilleri et Izzo, Apostolidis, Markaris et Martinidis font en effet rarement l'éloge de la gastronomie méditerranéenne dans leurs romans, et ce en dépit de certaines références à la cuisine grecque ou constantinopolitaine ${ }^{45}$. On pourrait s'interroger sur cette absence de « promotion culinaire » dans le roman policier grec. À mon sens, celle-ci est sans doute liée au fait que le nouveau roman policier hellénique tourne à la satire de la Grèce contemporaine. Ce point est particulièrement évident dans la relation de l'enquêteur grec à la nourriture. Contrairement à ses homologues espagnol, italien ou français, l'investigateur grec n'apparaît jamais sous les traits d'un fin gourmet qui apprécie la cuisine de son terroir. C'est un provincial ${ }^{46}$ un peu rustre qui mange un peu tout et n'importe quoi, surtout quand les repas sont gratuits (Martinidis). De manière plus générale, cet article nous a permis de voir que la cuisine échappe dans le roman policier grec à un simple effet de "couleur locale». Si, dans ses thrillers, Pétros Martinidis parodie l'antique tradition du symposion pour se moquer des koultouriarides, les intellectuels (de province), Pétros Markaris dote la gastronomie 
d'une dimension symbolique. En mettant en scène un enquêteur regrettant la cuisine grecque d'autrefois et raffolant des légumes farcis mitonnés par son épouse, l'auteur athénien se livre à une investigation sur la société hellénique contemporaine, une société arrimée à ses traditions, où les tâches domestiques sont encore clairement réparties au sein du couple (l'homme travaille, la femme cuisine). De manière plus audacieuse, on pourrait affirmer que la présence de la cuisine s'inscrit dans un roman policier qui affiche de plus en plus clairement sa dimension politique. Contrairement aux romans policiers apolitiques de Yannis Maris, ce n'est pas, en effet, à l'anatomie d'un crime que l'on assiste dans les « polars » de Markaris et de ses homologues, mais à l'anatomie d'un pays nommé Grèce. La présence symbolique de la cuisine s'intègre parfaitement selon nous dans cette réflexion politique effectuée par le nouveau roman policier grec.

\section{BIBLIOGRAPHIE}

COURTINE Robert, 2013, Simenon et Maigret passent à table : les plaisirs gourmands de Simenon \& les bonnes recettes de Madame Maigret, Paris : La Table Ronde, « La petite vermillon ».

MARCOU Loïc, (à paraître), Le roman policier grec (1935-2015) : essai sur les enjeux d'un genre

(para)littéraire en Grèce, Paris : Classiques-Garnier, « Perspectives comparatistes ».

TONNET Henri, 2007, « Le roman policier en Grèce, de 1995 à aujourd'hui », Revue des études néohelléniques, $\mathrm{n}^{\circ} 3$, Paris/Athènes, p. 127-145.

TONNET Henri, 2009, « Le roman policier grec des origines à 2008 », in « La Grèce et le polar ", revue Desmos, $\mathrm{n}^{\circ}$ 30, Paris, p. 11-21.

TONNET Henri, 2010, « Le roman policier grec d'hier et d'aujourd'hui », in « La Grèce, une utopie », revue Siècle 21, $\mathrm{n}^{\circ}$ 17, Paris, p. 13-16.

VÁZQUEZ MONTALBÁN Manuel, 2003, les Recettes de Carvalho, Paris : Christian Bourgois, « Littérature étrangère ».

VUILLERMET Maryse, 2005, « Boire et manger chez Jean-Claude Izzo ou la nourriture des justes », in Mireille PIAROTAS \& Pierre CHARRETON (dir.), le Populaire à table : le Boire et le Manger aux XIX et XX $x^{e}$ siècles, Saint-Étienne : Publications de l’Université de Saint-Étienne, p. 275-284.

\section{NOTES}

1. Cette expression argotique, que l'on retrouve dans maints romans policiers de la Série Noire de Gallimard, est sans doute née par référence aux personnes placées en garde à vue, qui ne peuvent manger que si elles passent aux aveux. Sur ce point, voir entre autres Albert DollLon, Dictionnaire de l'argot, Paris : Robert Laffont, « Bouquins », 2010. 
2. C'est ce que rappelle notamment Pétros MARKARIS dans $K \alpha \tau^{\prime} \varepsilon \xi \alpha \kappa o \lambda o v ́ \theta \eta \sigma \eta$, Athènes :

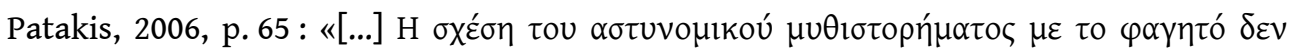

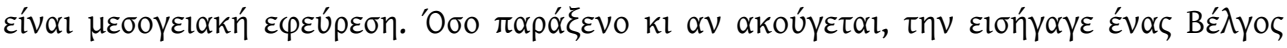

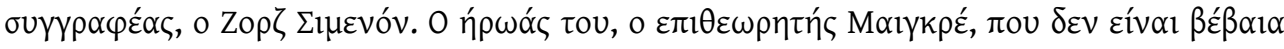

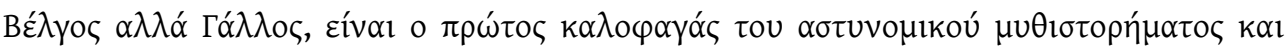

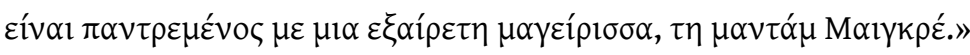

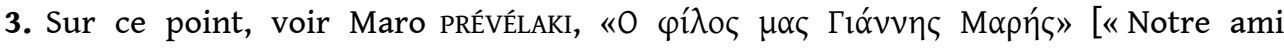
Yannis Maris »], in la Littérature de l'après-guerre. Thématiques et formes d'écriture, Paris : Inalco, 1992, p. 93-103.

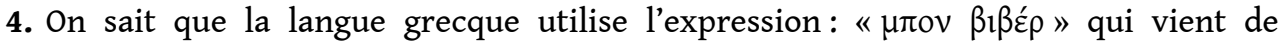
l'anglais et qui est une déformation de l'expression française : « bon vivant ».

5. Personnage de détective en fauteuil né sous la plume du romancier américain Rex Stout (1886-1975) et apparaissant dans un grand nombre de romans et nouvelles des années trente aux années soixante-dix. Rappelons succinctement que Nero Wolfe pèse 130 kilos et qu'il a deux passions dans la vie : les orchidées et la gastronomie. Ses connaissances culinaires et œnologiques lui permettent de donner des conférences en gastronomie.

6. Maryse VUILLERMET, «Boire et manger chez Jean-Claude Izzo ou la nourriture des justes ", in le Populaire à table : le Boire et le Manger aux XIX et XXe siècles (sous la direction de Mireille PIAROTAS et Pierre CHARRETON), Saint-Étienne : Publications de l'Université de Saint-Étienne, 2005, p. 275-284.

7. Jean-Claude Izzo, Chourmo, Paris : Gallimard, « Folio », 1996, p. 133.

8. Il s'agit de la femme médecin légiste Kay Scarpetta, qui apparaît pour la première fois dans le roman Postmortem (1990). Rappelons que Patricia Cornwell et Marlene Brown ont écrit un ouvrage de cuisine inspiré des recettes de Kay Scarpetta : Crimes et délices. La Cuisine de Kay Scarpetta, Paris : Calmann-Lévy, 2002.

9. Il convient de ne pas oublier le commissaire Brunetti de Donna Leon, qui est lui aussi une «bonne fourchette».

10. François RIVIÈRE \& Anne MARTINETTI, Crèmes et Châtiments, recettes délicieuses et criminelles d'Agatha Christie, Paris : Le Masque, « Grands formats », 2010.

11. François RIVIÈRE \& Anne MARTINETTI, la Sauce était presque parfaite, 80 recettes d'après Alfred Hitchcock, Paris : Cahiers du Cinéma, «Beaux livres », 2008.

12. Anne MARTINETTI, Alimentaire, mon cher Watson! Paris : Chêne, 2010.

13. Arlette LAUTERBACH \& Alain RAYBAUD, le Livre de cuisine de la Série Noire, Paris : Gallimard, "Série Noire hors-série ", 1999. Voir aussi des mêmes auteurs : le Livre des alcools de la Série Noire, Paris : Gallimard, « Série Noire hors-série », 2001.

14. Manuel VÁzqueZ montalbán, les Recettes de Carvalho, Paris : Christian Bourgois, "Littérature étrangère », 2003.

15. Robert COURTINE, Simenon et Maigret passent à table. Les plaisirs gourmands de Simenon \& les bonnes recettes de Madame Maigret, Paris : La Table Ronde, "La petite vermillon", 2013.

16. Même si l'appellation "polar méditerranéen » semble plus relever d'une étiquette éditoriale (donc d'une stratégie commerciale) que d'un véritable (sous-)genre littéraire, cette expression est de plus en plus utilisée pour désigner des romans policiers dont 
l'action se déroule dans l'Europe du sud par opposition au "polar nordique». Dénonçant les dérives des sociétés d'Europe méridionale tout en mettant en valeur leur

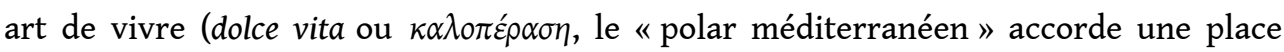
importante à la cuisine, comme le montrent notamment les récits de Vázquez Montalbán, Camilleri et Izzo.

17. Nous reprenons ici la formule employée par le professeur Henri Tonnet dans son article : "Le roman policier en Grèce, de 1995 à aujourd'hui ", Revue des études néohelléniques, $\mathrm{n}^{\circ}$ 3, Paris/Athènes, p. 127-145.

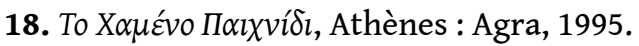

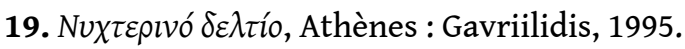

20. Sur ce point, on se reportera aux remarques de Pétros MARKARIs dans

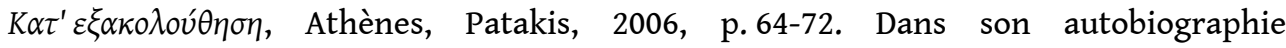
intellectuelle, Markaris souligne les différences entre roman policier méditerranéen et polar nordique. Dans ce dernier type de roman policier, selon Markaris, les enquêteurs ne sont jamais présentés comme des gastronomes avertis, contrairement à leurs homologues d'Europe du sud (Carvalho, Montalbano, Montale).

21. Cette expression désigne les trois romans policiers dans lesquels Jean-Claude Izzo met en scène le policier marseillais Fabio Montale : Total Khéops (1995), Chourmo (1996), Soléa (1998).

22. Manuel Vázquez montalbán, Tatouage. Extrait cité in les Recettes de Carvalho, Paris : Christian Bourgois, 1996, p. 10-11.

23. Parfois flanqué du journaliste Makris, l'avocat Ikonomou apparaît dans plusieurs

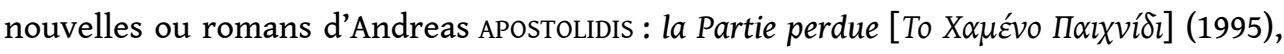

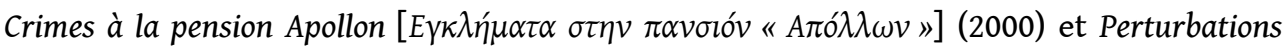

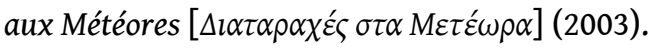

24. On ne trouve en effet que quelques allusions à la gastronomie hellénique dans

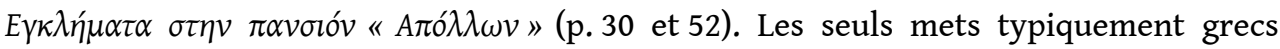

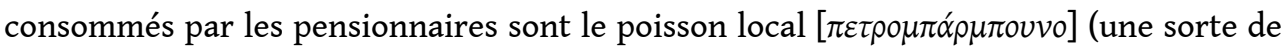

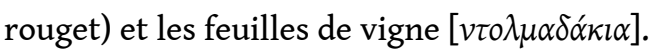

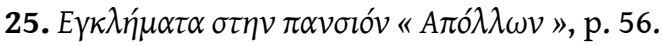

26. Ibid., p. 77, 82 et 246 .

27. Ibid., p. 96.

28. Ibid., p. 115, 144, 218 et 225 .

29. Les seules boissons typiquement grecques que boivent les clients de la pension

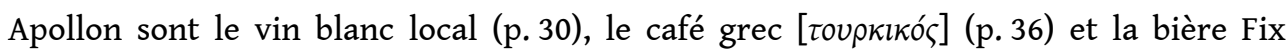
(p. 232).

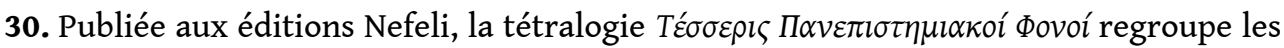

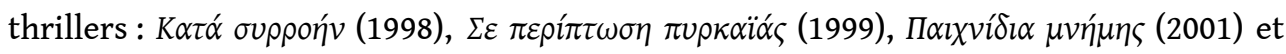

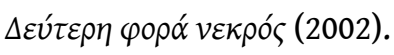

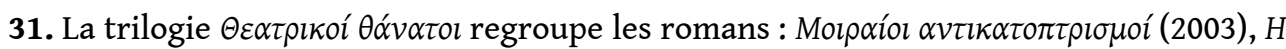

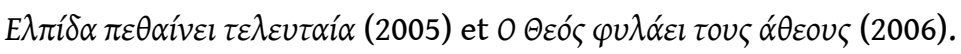

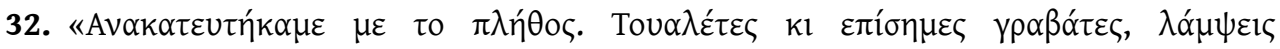

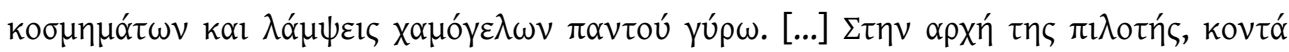

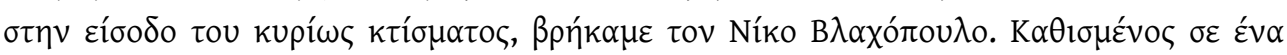




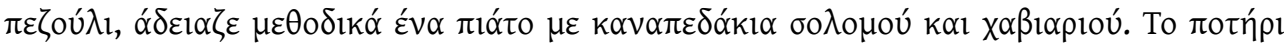

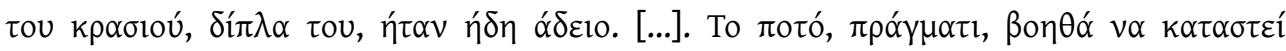

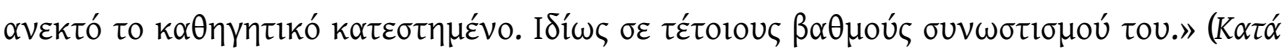
бuppớv, p. 161, 162, 163 et 165).

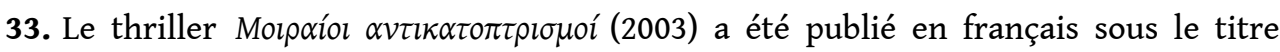
Reflets du destin, Paris : L'Harmattan, « Lettres grecques », 2013 (trad. de Henri Tonnet).

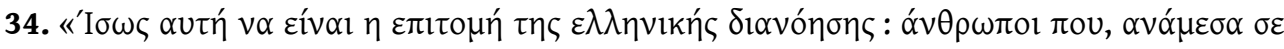

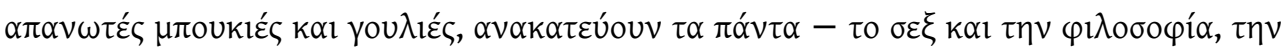

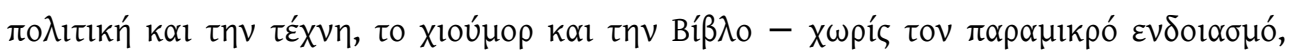

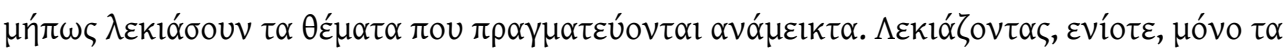

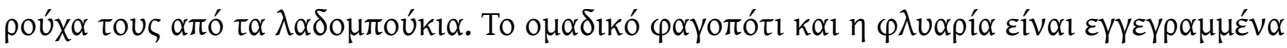

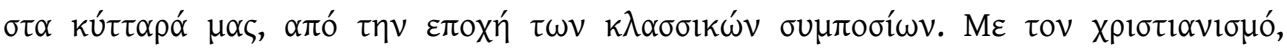

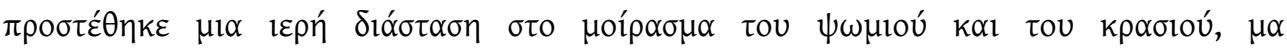

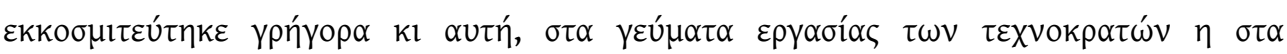

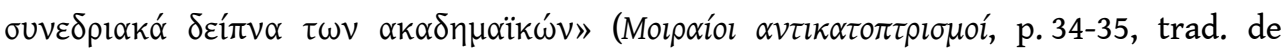
Henri Tonnet).

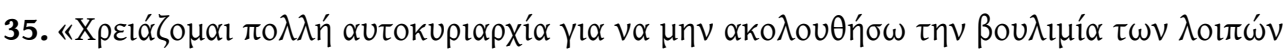

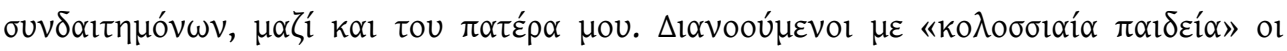

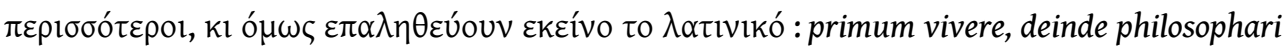

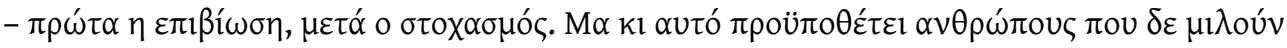

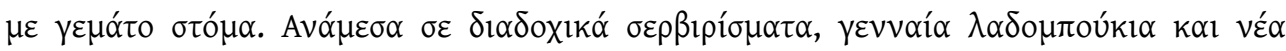

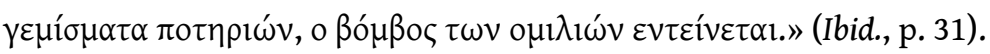

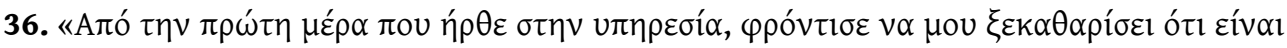

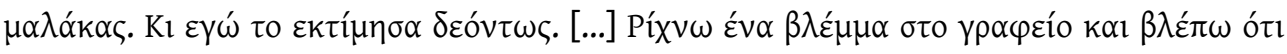

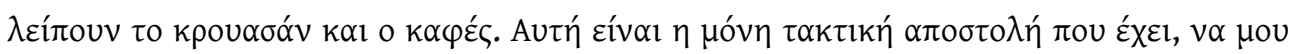

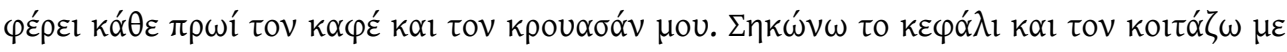

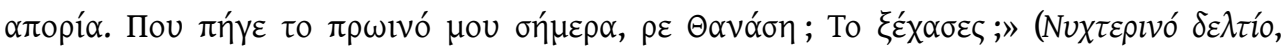
p. 9-10).

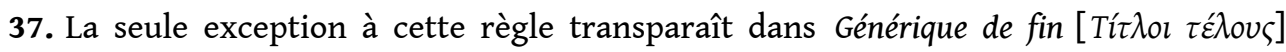
(2014), le dernier roman policier de Markaris. En raison de la crise financière qui continue à sévir en Grèce, Adriani est en effet contrainte d'avoir recours aux services et à la générosité de sa fille Katerina, de son gendre Phanis Ouzounidis et même de Lambros Zissis (l'ancien communiste devenu l'ami de son policier de mari) lorsqu'elle organise de grands repas familiaux. C'est dire si la «crise grecque» modifie les habitudes culinaires du couple Charitos...

38. Le "patriarche » est le surnom affectueux donné par Pétros Markaris au pionnier de la littérature policière grecque, Yannis Maris (1916-1979), dans un colloque organisé à Athènes en avril 2011.

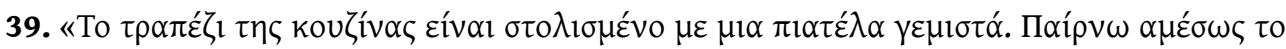

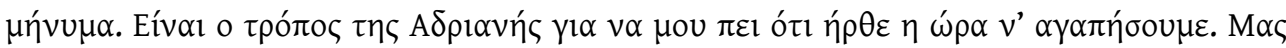

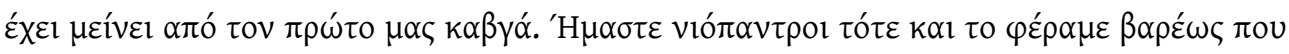

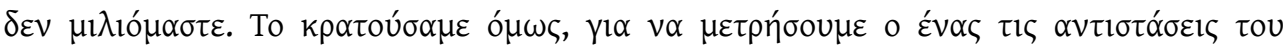

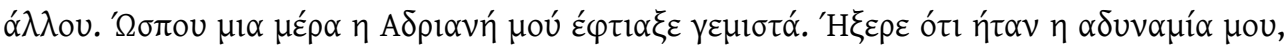

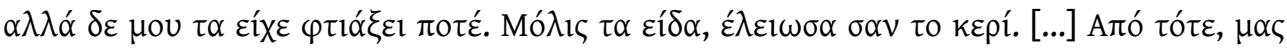




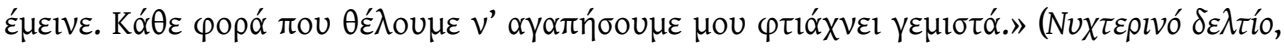
p. 225).

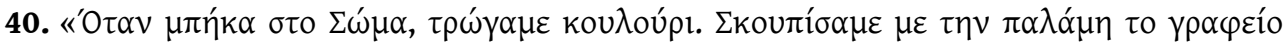

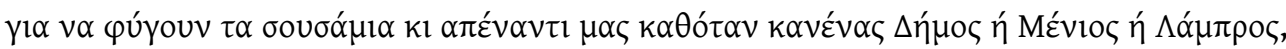

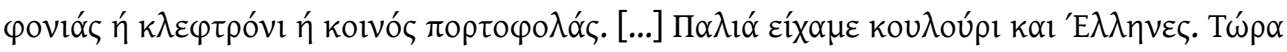

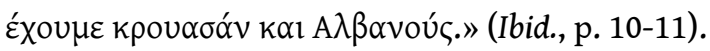

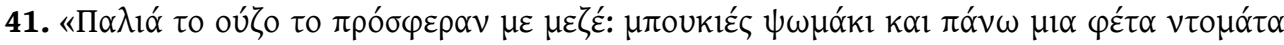

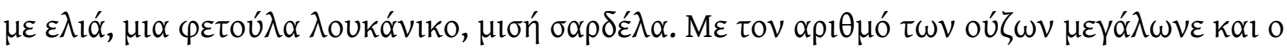

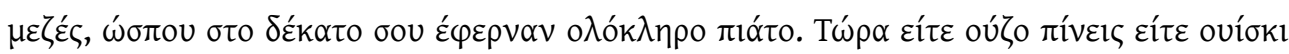

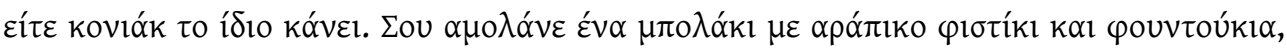

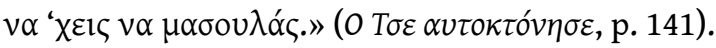

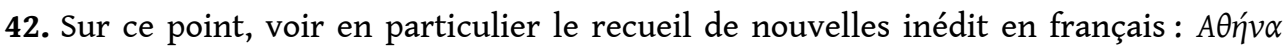
$\pi \rho \omega \tau \varepsilon v ́ o v \sigma \alpha \tau \omega v$ B $\alpha \lambda \kappa \alpha v i ́ \omega v$, Athènes : Gavriilidis, 2004.

43. Rappelons que des membres du parti néo-nazi Aube dorée agressent Katerina, la

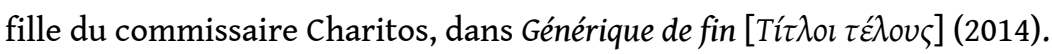

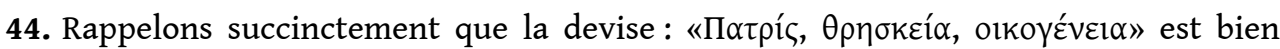

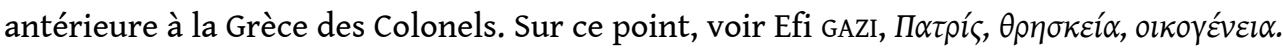

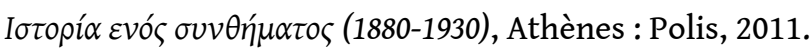

45. Comme le souligne Markaris, les plats préparés par Adriani, l'épouse du commissaire Charitos, sont d'origine constantinopolitaine ou micrasiatique. Sur ce point, voir $K \alpha \tau^{\prime} \varepsilon \xi \alpha \kappa o \lambda o v ́ \theta \eta \sigma \eta$, éd. cit., p. 68.

46. N'oublions pas que le commissaire Charitos de Markaris est originaire de Konitsa, une petite ville épirote située près de la frontière albanaise. Sur ce point, voir $\Pi \alpha \lambda \lambda_{1}$, $\pi \circ \lambda v^{\prime} \pi \alpha \lambda_{i \alpha}$, p. 44.

\section{RÉSUMÉS}

Dans cet article, je mettrai en exergue la relation unissant l'enquêteur avec la cuisine dans la littérature policière. Après un bref rappel sur la place de la gastronomie dans la littérature policière en général, je me focaliserai sur le « nouveau roman policier grec » (notamment sur les romans et nouvelles d'Andréas Apostolidis, Pétros Markaris et Pétros Martinidis) en montrant les points de rapprochement - ou de divergence - entre roman policier hellénique et «polar méditerranéen ».

In this article, I will intend to show the long-standing relationship in most crime fiction detectives' lives-that is with food and gastronomy. I will focus on recent Greek crime fiction (especially the novels or short stories of Andreas Apostolidis, Pétros Markaris and Pétros Martinidis) and will compare the role of food in Greek detective fiction with its place in the Mediterranean Noir.

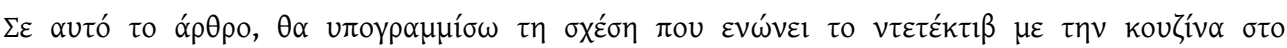

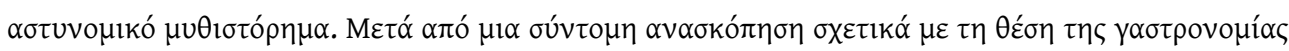




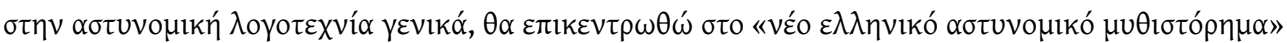

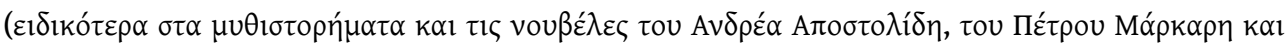

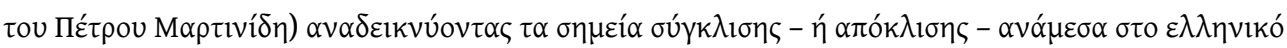

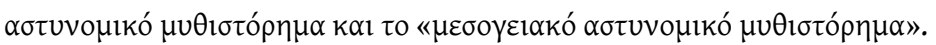

\section{INDEX}

Index géographique : Grèce

motsclesmk АПОСТОЛИДИС АНДРЕАС (1953-), КАМИЛЕРИ АНДРЕА (1925-), ГАСТРОНОМИЈАТА, ИЗЗО ЖАН КЛОД (1945-2000), МАРКАРИС ПЕТРОС (1937), МАРТИНИДИС ПЕТРОС (1946-), МОНТАЛБАН ВАСКЕЊ МАНУИЛ (1939-2003), МОДЕРНОСТА, ТРАДИЦИЈА, ГРЦИЈА, ДВАЕСЕТТИОТ ВЕК, ДВАЕСЕТ И ПРВИОТ ВЕК, ДЕТЕКТИВСКИ РОМАНИ

Keywords : Apostolidis Andreas (1953-), Camilleri Andrea (1925-), Gastronomy, Izzo Jean-Claude (1945-2000), Markaris Petros (1937-), Martinidis Petros (1946-), Montalbán Vasquez Manuel (1939-2003), Modernity, Tradition, Greece, Twentieth century, Twenty First century, Detective novels

Mots-clés : Apostolidis Andreas (1953-), Camilleri Andrea (1925-), Camilleri Andrea (1925-), gastronomie, Izzo Jean-Claude (1945-2000), Izzo Jean-Claude (1945-2000), Markaris Pétros (1937-), Markaris Pétros (1937-), Martinidis Pétros (1946-), Martinidis Pétros (1946-), Montalbán Vasquez Manuel (1939-2003), Montalbán Vasquez Manuel (1939-2003), modernité, tradition motsclestr Apostolidis Andreas (1953-), Camilleri Andrea (1925-), Gastronomi, Izzo Jean-Claude (1945-2000), Markaris Petros (1937-), Martinidis Petros (1946-), Montalbán Vasquez Manuel (1939-2003), Modernlik, Gelenek, Yunanistan, Yirminci yüzyıl, Yirmi birinci yüzyıl, Dedektif romanları

Thèmes : Littérature policière

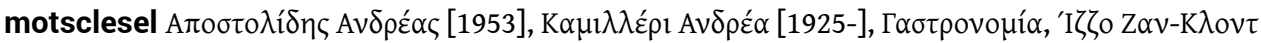

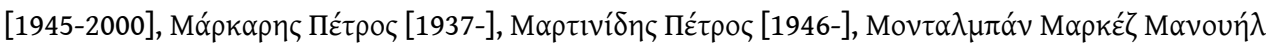

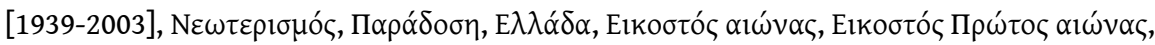

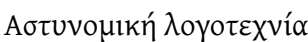

Index chronologique : vingt-et-unième siècle

\section{AUTEUR \\ LOÏC MARCOU}

CREE-EA4513, Inalco, USPC 